\title{
A Systematic Review of Cancer of Unknown Primary in the Head and Neck Region
}

\section{Milosz Pinkiewicz (D) Karolina Dorobisz (D) \\ Tomasz Zatoński}

Department of Otolaryngology, Head and Neck Surgery, Wroclaw Medical University, Wrocław, Poland
Correspondence: Karolina Dorobisz Department of Otolaryngology, Head and Neck Surgery, Wroclaw Medical University, Borowska 213, Wroclaw, 50-529, Poland

Email dorobiszkarolina@gmail.com
Background: Despite the vivid progress in molecular and genetic profiling, extensive diagnosis and multiple therapeutic modalities, cancers of unknown primary in the head and neck region continue to be a formidable challenge.

Aim: The purpose of the review is to present the most recent and well-established findings concerning cancers of unknown primary (CUPs) in the head and neck patients and consequently to provide medical specialists with essential information regarding the biology, pathology, histology, diagnosis and treatment of CUP in the head and neck region.

Material and Methods: The Medline/PubMed database was searched by using the following keywords "CUP", "cancer of unknown primary", "CUP biology", "head and neck". The references of the publications of interest were also screened for relevant papers. There were no limitations in regard to the publication date.

Conclusion: Unique biology and pathophysiology prompt the management of CUP to be demanding. The vivid rise in HPV-related CUPs during the last 20 years has put more focus on this phenomenon. New findings concerning the enigmatic biology of CUP provide fundaments for targeted therapy. Despite the availability of various diagnostic methods, the diagnosis of CUP continues to be a time-consuming, strenuous process that eventually provides answers. It remains controversial as to what combination of treatment methods is the most effective. There is no consensus on the value of combining chemotherapy with radiotherapy. Highly specific surgical treatments for particular histological types of CUP produce more satisfactory results. It is paramount to establish reliable guidelines concerning the diagnosis and treatment of CUP patients.

Keywords: cancers of unknown primary in the head and neck region, diagnosis of cancers of unknown primary, treatment of cancers of unknown primary, head and neck oncology

\section{Introduction}

Cancer of unknown primary is a malignant metastatic cancer whose primary site remains to be undetermined after extensive work-up, which combines comprehensive clinical, radiographic, and surgical evaluation including biopsies with subsequent resection of suspicious primary sites. ${ }^{1}$ On the global scale, the CUP is ranked as sixth to eighth most common cancer. The CUP prevalence in the head and neck is considered to be around $2-5 \%$ of all HNSCC cases. ${ }^{2}$ The most encountered site of primary origin is oropharynx (particularly the crypt of the epithelium of the palatine tonsils and base of the tongue or lingual tonsil). ${ }^{1}$ CUP of the oropharynx is known for metastasis to levels II or III, in certain cases to levels IV as well. ${ }^{1}$ The most prevalent histotype is adenocarcinoma, followed by undifferentiated carcinomas such as melanoma or lymphoma. ${ }^{3}$ The signs and symptoms of CUP may differ 
considerably and depend mostly on the location of metastasis. The primary site is eventually found in $60 \%$ of patients, despite the exhaustive diagnostic process. ${ }^{1}$ Stella et al have noted that $80-85 \%$ of patients present aggressive potential and unpredictable patterns of metastatic spread prompting the diagnosis to be poor - median survival of 4-12 months. ${ }^{4}$ Furthermore, research shows that CUP survivors are more prone to develop subsequent cancers. ${ }^{4}$ The optimal treatment strategies for HNCUP remain controversial as there is a vivid lack of randomized trials. For the moment, the golden standard of therapy for cancers of unknown primary in the head and neck region involves (chemo) radiation therapy (RT) alone or in combination with surgery. Nonetheless, the exact information regarding the sequence, the extent of irradiated volumes, and the optimal curative RT dose remain to be controversial. $^{5}$ Even though intensity-modulated RT (IMRT) allows obtaining excellent rates of nodal control and disease-free survival, nodal and mucosal recurrences continue to occur ${ }^{5}$ Although chemotherapy is often used together with radiotherapy, it remains ambiguous whether it adds any value to the treatment. ${ }^{6}$

\section{Review}

\section{Biology and Pathophysiology}

The pathogenesis of CUP is a multi-step process involving clonal proliferation, invasion and intravasation of cancer cells from the primary tumour, widespread dissemination via circulation, extravasation in various organs and unconcealed colonisation at metastatic sites. ${ }^{7,8}$ This sequence is prompted by the multiple interdependent changes occurring in cells. Amongst them are limitless replicative potential, evasion of apoptosis and immune destruction, chromosomal alterations, reprogramming of energy metabolism, tissue invasion and metastasis, resistance to growth-inhibitory signals and self-sufficiency in growth signals. According to Rassy et al, the expression of p53 is reported in $48-70 \%$ (overexpression in 53\%) of patients with CUP. ${ }^{8}$ Vascular endothelial growth factor-A (VEGF-A) is expressed in 26$83 \%$ of CUP patients. $^{9-12}$ Karavasilis reports that Metalloproteinase (MMP)-2 and MMP-9 are expressed in $69 \%$ (overexpression in $49 \%$ ) and $49 \%$ (overexpression in $36 \%$ ) of patients with CUP, respectively. ${ }^{12}$ Gatalica et al have shown that PD-1 expression in the tumour-infiltrating lymphocytes was present in $58.7 \%$ of patients with CUP, while PD-L1 expression was observed in $22.5 \%$ of cancer cells in tumours, respectively. ${ }^{13}$ Reportedly, the expression of phosphorylated AKT (pAKT) was detected in $73.2 \%$ of patients with CUP and is correlating with worse survival. ${ }^{14}$ Hedley et al have noted that almost $70 \%$ of patients with CUP display an increase in transcripts for proteins that are involved in the process of DNA damaging as well as function in homologous recombination repair networks, such as BRCA1, ATM and CHEK2 (checkpoint kinase 2) - this has prompted authors to presume that CUP is chromosomally unstable. $^{15}$ It is known that CUP is characterized by uncommon spreading and clinical aspects, which are inconsistent with the supposed site of origin. ${ }^{3}$ The primary hypothesis considering CUP biology implies that the neoplasm could result from a stem cell, without any prior premalignant lesion or a primary tumor, whereas the second indicates that CUP represents the rapid progression of metastasis from a very early primary tumor. ${ }^{3}$ Natoli et al have reported several chromosomal abnormalities concerning CUPs, with the aberration of chromosomes 1 , 6,7 , and 11 being the most common ones. ${ }^{16}$ Stella et al have noted that $70 \%$ of adenocarcinomas of the unknown primary had underlying aneuploidy. ${ }^{4}$ Pantou et al have identified other chromosome modifications such as 1q21, 3p13, 6q15-23, 7q22, 11p12-5 and 11q14-24.51. ${ }^{17}$ Preferential involvement of 4q31, 6q15, 10q25 and 13q22 has been reported in adenocarcinomas, while other carcinomas have a $11 \mathrm{q} 22$ involvement. $^{8}$

\section{Histology}

Numerous studies have concluded that squamous cell carcinoma is the most frequently found histological type in HNCUP patients, following this was undifferentiated carcinoma and adenocarcinoma. ${ }^{2,3,18}$ However, a large study of 2935 patients with CUP in Zurich found adenocarcinomas to be the most abundant histological group (42.3\%). ${ }^{19}$ Stella et al have also concluded that adenocarcinomas are the most common histological group. ${ }^{4} \mathrm{~F}$ Losa reports that adenocarcinomas and undifferentiated tumours have a more unfavourable prognosis (3.5\% 3-year survival) than squamous cell carcinomas (41.6\% 3-year survival). ${ }^{3}$

\section{Risk Factors}

Risk factors involve heavy smoking (26 or more cigarettes/day) and HPV infection. Alcohol consumption is of little relevance. ${ }^{20-22}$

\section{HPV-Related CUP}

Although HPV-related oropharyngeal cancer has been present for many years, the prevalence has progressed during 
the last 20 years. ${ }^{23}$ Several studies have concluded that human papillomavirus (HPV) is the most significant biomarker for disease-free survival and overall survival in patients suffering from oropharyngeal cancer. ${ }^{21,23}$ It is generally known that HPV-related oropharyngeal SCCs metastasize early in the development of the disease, while the primary tumor is still insignificant in size. ${ }^{23}$ Cystic degeneration is oftentimes observed in metastases from HPV-related SCC. ${ }^{1}$ Few retrospective studies have shown that patients with HPV-positive tumors have a more favourable prognosis than patients with HPV-negative tumors. $^{21,23}$ In a study of 743 patients, K. Kian Ang et al have reported that HPV-positive oropharyngeal cancer was more prevalent amongst patients who had never smoked and those with a lower number of cumulative pack-years of tobacco smoking than amongst heavy smokers. Furthermore, HPV-positive oropharyngeal cancer was significantly associated with few favourable prognostic factors such as age, white race, better performance status, absence of anemia, and smaller primary tumors. ${ }^{21}$

\section{Diagnostic Methods}

A comprehensive diagnosis has to involve a full case history, physical as well as hematological examination. Subsequently, mucosal sites of the upper aerodigestive tract have to be evaluated with the use of endoscopy. An endoscopic examination may include fiber optic laryngoscopy, bronchoscopy, esophagogastroscopy or direct nasopharyngoscopy. ${ }^{18}$ Following that, an MRI of the head, neck and chest regions is recommended, together with a contrast-enhanced CT of the chest, abdomen and pelvic cavity. Upper gastroenterography may also be included. ${ }^{24}$ The valid role of additional bone scan and 18-F-FDG-PET has been highlighted by numerous studies. ${ }^{18}$ Qaseem et al have shown that Positron emission tomography-computed tomography (PET/CT) and multiparametric 3T-MRI (MP-MRI) provide comparable precision during the diagnosis of CUP with metastasis in neck lymph nodes. ${ }^{20}$ PET characterizes with average accuracy concerning anatomical sites or functional irregularities due to low tracer uptake by certain tumor tissues. Thus, it is recommended to combine PET with CT. The prospective study carried out by Moller has found that PET/CT is not superior to $\mathrm{CT}^{25}$ Consequently, PET is not recommended during initial testing but may be used for specific clinical presentations such as single metastases or cervical lymphadenopathies. ${ }^{3}$ In women, mammography should also be performed. In case the result is equivocal, magnetic resonance imaging should also be implemented. ${ }^{3}$ There are present commercial tests with gene expression-based assays that allow diagnosis of tumors of unknown or uncertain origin such as biotheranostics (RT-PCR for mRNA), Pathwork [microarray for messenger RNA (mRNA)] and Rosetta Genomics and Prometheus (RT-PCR for microRNA). ${ }^{4}$ The aforementioned tests declare prognostication correctness in known primary cancers between $80 \%$ and $90 \%{ }^{4}$ Chernock states that primary cancers occurring in salivary glands may mimic metastases of unknown primary and have to be differentiated from cutaneous metastases to the parotid gland, a subset of which may not have a clinically apparent primary skin tumor. ${ }^{1}$

\section{Treatment}

Available methods of treatment include surgery, chemotherapy and radiotherapy. Decisions regarding CUP treatment should be reached within a multidisciplinary team and with consideration of such factors as the patient's overall condition, potential treatment-related toxicity, distribution in the neck and future possibilities of rehabilitation. ${ }^{14}$ Furthermore, it is crucial to distinguish between two main groups of CUPs. The first one considers CUPs of which origin is hidden and clinical, as well as pathological characteristics, do not indicate any sites and the second group concerns CUPs whose primary site of the tumor is significantly suggested by clinical and pathological characteristics ${ }^{18}$. The purpose of concomitant chemoradiotherapy in CUP patients is debatable since there is no clinical evidence due to the lack of prospective studies. Treatment and outcomes of treating cancer of unknown primary in the head and neck region are summarized in Table 1.

\section{Surgery}

Possible surgical procedures involve neck dissections (bilateral, unilateral, radical or modified radical neck dissection, depending on the location of the lesion) and tonsillectomy. Neck dissection is not always necessary, for example, in N1 disease. ${ }^{4}$ ASCO guidelines imply that ipsilateral palatine tonsillectomy should be implemented in patients who present unilateral lymphadenopathy and whose primary site could not be confirmed during the initial evaluation. ${ }^{26}$ Were the palatine tonsillectomy to fail in identifying the primary site, an ipsilateral lingual tonsillectomy should be planned. ${ }^{26}$ Bilateral thyroidectomy is recommended in the case of diagnosing metastatic 


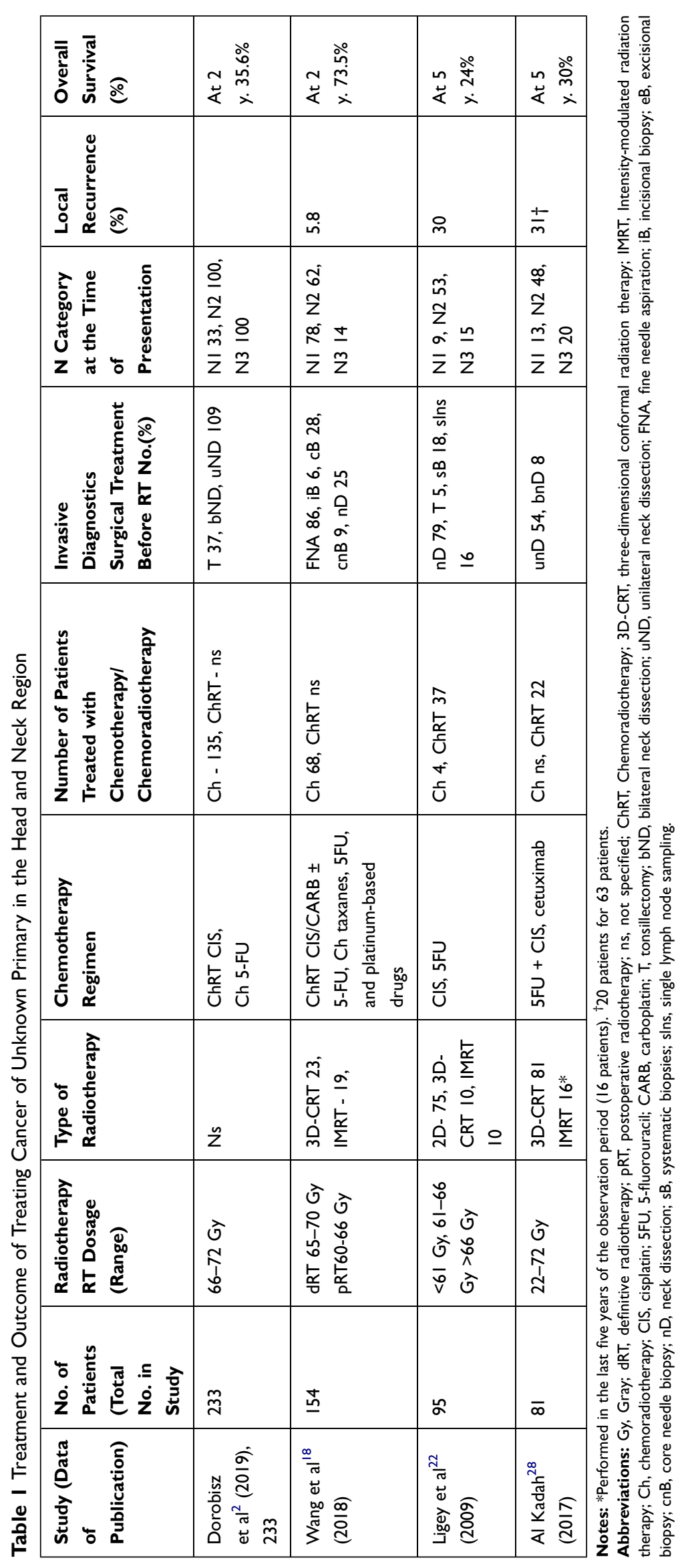


adenocarcinoma. In the case of bilateral lymphadenopathy and no confirmation of a primary site during endoscopy, it is recommended to perform a unilateral lingual tonsillectomy on the more influenced side. ${ }^{26} \mathrm{~A}$ contralateral lingual tonsillectomy may follow in case the ipsilateral procedure will not identify a primary. ${ }^{26}$ Even though there is a lack of randomized trials that have compared the efficiency of performing ipsilateral tonsillectomy, research shows that the yield for the localization of the primary rises to a $30 \%$ to $50 \%$ range. $^{26}$

ASCO guidelines highlight that bilateral palatine tonsillectomy should be avoided after bilateral lingual tonsillectomy. ${ }^{26} \mathrm{~A}$ paper published by Dorobisz et al shows that there is a statistically important correlation between surgical treatment and survival $(\mathrm{P}<0.001)$. Reportedly, the survival rate of 12 months in surgical patients is approximately three times higher than in patients who did not undergo surgical treatment. ${ }^{2}$ The $\mathrm{N}$ adjustment has increased it more than six times. Surgically treated patients had a two and a half times higher chance of survival than non-surgically treated patients. $^{2}$ Nonetheless, there is no consensus on the efficacy of neck dissection. Literature does not indicate that neck dissection improves the outcome. ${ }^{5}$

\section{Chemotherapy}

A staggering amount of studies highlights that CUPs are resistant to chemotherapy, prompting this form of treatment to be ineffective, especially concerning long-term survival. $^{1,4,18,27,28}$ There is no preferred combination of drugs used in chemotherapy. ${ }^{4}$ The platinum-based combination is used as the first-line approach to CUP treatment and because of the lower toxicity of carboplatin as compared to cisplatin, the favoured regimen is carboplatin/ paclitaxel. ${ }^{29}$ Such combinations have allowed achieving response rates of $30-40 \%$ and a 2 -year survival rate of $20-25 \%$ can be accomplished if applied as first-line therapy. ${ }^{30}$ The efficacy and toxicity of the combination of paclitaxel, carboplatin, bevacizumab, and erlotinib in the first-line treatment of CUP patients have been assessed in a lately conducted Phase II trial. ${ }^{29}$ Recruited patients received the aforementioned drugs and treatment cycles were redone every 21 days. Bevacizumab-erlotinib treatment was implemented until tumor progression, once the four cycles of paclitaxel and carboplatin were abandoned. ${ }^{29}$ This combination of drugs led to a median progression-free survival time of 8 months, whereas $38 \%$ of patients were free of progression at 1 year. Hainsworth et al reported that the median survival time and 2-year overall survival rates were 12.6 months and $27 \%$, respectively; the toxicity profile was well tolerated. ${ }^{29}$ It is still ambiguous whether second-line therapy can impact the survival of patients with CUP. It has been noticed that patients who have shown favourable responses to firstline chemotherapy might also benefit from second-line chemotherapy. ${ }^{4}$

\section{Radiotherapy}

Radiotherapy treatment (RTT) is a valuable tool in CUP therapy. The irradiated areas mostly involve the hypopharynx, the base of the tongue, the nasopharynx and tonsillar fossa or the unilateral or bilateral neck. ${ }^{18} \mathrm{Al}$ Kadah described using a standard fractionation with single doses of 1.8-2 Gy under the ICRU specifications once a day over 6-8 weeks resulting in total doses ranging from 22 to 72 Gy, mainly 50-72 Gy was applied. ${ }^{28}$ Dorobisz et al reported treating 165 patients with a standard dose of 2 Gy, 5 times a week and noticed a statistically significant link between radiotherapy and survival $(\mathrm{P}<0.001)-$ the survival rate of 12 months in patients who underwent radiation therapy was almost seven times higher than in subjects treated differently $(\mathrm{OR}=6.80)$ and the survival rate of 24 months was four and a half times higher $(\mathrm{OR}=4.47){ }^{2}$ Wang et al report that radiotherapy to pharyngeal mucosa was associated with improved OS and PFS in their study of 42 patients who underwent RTT. ${ }^{18}$ There are no reported differences in OS and disease-free survival in CUP patients between those who received ipsilateral radiotherapy and those who received comprehensive radiotherapy (including the potential mucosa and ipsilateral or bilateral neck). ${ }^{18}$ By the same token, Ligey et al have reported in their retrospective study which compared bilateral neck radiotherapy with unilateral neck radiotherapy that there is no significant difference regarding locoregional control and survival since the 5-year OS rates were $23 \%$ after bilateral radiotherapy $(\mathrm{P}=0.944)$ and $22 \%$ after unilateral radiotherapy. ${ }^{22}$ This is contrary to Grau et al who have shown in the largest series on CUP that bilateral treatment together with the entire pharyngolaryngeal mucosa allowed obtaining lower rates of disease recurrence. ${ }^{31}$ Furthermore, the authors have pointed out that bilateral radiation is associated with better survival expectations than those provided by the radiation limited to the involved side of the neck. ${ }^{31}$ Studies have clearly shown that IMRT provides excellent results for patients with cervical node squamous cell carcinoma metastases 
coming from an unknown head-and-neck primary tumour. Two studies reported survival rates at 5 years of $89 \%$ and $84 \%$. Primary chemoradiotherapy (CRT) is the recommended treatment option in patients with an unresectable bulk or incomplete resection since it allows avoiding excessive toxicity from surgery and postoperative chemoradiotherapy. ${ }^{6}$

\section{Limitations of This Study}

Authors have strived to create a highly succinct yet comprehensive systematic review.

Such an approach demands a rigorous selection of articles. Although authors have aimed to gather all fundamental aspects as well as unique insights, there are surely some experiences and information, which have not been included due to their little significance. Some have been omitted as they did not add anything illuminating. These conclusions have been drawn by the authors. Thus, the selection may be questioned.

\section{Conclusions}

Despite the available knowledge and the overall interest in medical society CUPs continue to baffle medical practitioners. Current guidelines fail to provide clear recommendations regarding the most effective combination of therapeutic modalities. Furthermore, the details of radiotherapy, such as the range dose and the extent of the radiotherapy are also unresolved. Some authors find bilateral irradiation to be the most effective, whereas others stand by the treatment of only the involved side of the neck - the efficacy does not change, but the toxicity is smaller. Among numerous publications, one can observe an evident lack of consensus on the correct guidelines concerning diagnosis and management of CUP impede the care of CUP patients. There is a growing need for randomized clinical trials or prospective studies to compare the effectiveness of available treatments. The study carried out by Binder and others shows that the overall survival of CUP patients has not improved since early $1980 .^{15}$ The remaining hallmarks of CUP should be researched as the previous interest was focused on chromosomal instability and growth factor independence. Even though molecular diagnostics provided many answers in regard to the biology of CUP, it continues to fall short of providing survival benefits in clinical practice. ${ }^{8}$

\section{Disclosure}

The authors report no conflicts of interest in this work.

\section{References}

1. Chernock RD, Lewis JS. Approach to metastatic carcinoma of unknown primary in the head and neck: squamous cell carcinoma and beyond. Head Neck Pathol. 2015;9(1):6-15. doi:10.1007/s12105015-0616-2

2. Dorobisz K, Wlodarska-Polinska I, Pazdro-Zastawny K, et al. The impact of the patient's condition, diagnostic procedures and treatment on the survival of carcinoma of unknown primary site patients. Cancer Manag Res. 2019;11:6603-6614. Published 2019 Jul 16. doi:10.2147/CMAR.S204346

3. Losa F, Iglesias L, Pané M, et al. 2018 consensus statement by the Spanish society of pathology and the Spanish society of medical oncology on the diagnosis and treatment of cancer of unknown primary. Clin Transl Oncol. 2018;20(11):1361-1372. doi:10.1007/ s12094-018-1899-z

4. Stella GM, Senetta R, Cassenti A, Ronco M, Cassoni P. Cancers of unknown primary origin: current perspectives and future therapeutic strategies. J Transl Med. 2012;10(1):12. Published 2012 Jan 24. doi:10.1186/1479-5876-10-12

5. Kamal M, Mohamed ASR, Fuller CD, et al. Patterns of failure after intensity modulated radiation therapy in head and neck squamous cell carcinoma of unknown primary: implication of elective nodal and mucosal dose coverage. Adv Radiat Oncol. 2020;5(5):929-935. Published 2020 May 15. doi:10.1016/j.adro.2020.04.025

6. Müller von der Grün J, Tahtali A, Ghanaati S, Rödel C, Balermpas P. Diagnostic and treatment modalities for patients with cervical lymph node metastases of unknown primary site - current status and challenges. Radiat Oncol. 2017;12(1):82. Published 2017 May 10. doi:10.1186/s13014-017-0817-9

7. López-Lázaro M. The migration ability of stem cells can explain the existence of cancer of unknown primary site. Rethinking metastasis. Oncoscience. 2015;2(5):467-475. Published 2015 May 1. doi:10.18 632/oncoscience. 159

8. Rassy E, Assi T, Pavlidis N. Exploring the biological hallmarks of cancer of unknown primary: where do we stand today? Br J Cancer. 2020;122:1124-1132. doi:10.1038/s41416-019-0723-z

9. Rashid A, Hess KR, Lenzi R, et al. Overexpression and prevalence of molecular markers in patients with cancer of unknown primary (CUP). Proc Am Soc Clin Oncol. 2005;24:9683. n.d.Return to ref 57 in article. doi:10.1200/jco.2005.23.16_suppl.9683

10. van de Wouw AJ, Jansen RLH, Griffioen AW, Hillen HFP. Clinical and immunohistochemical analysis of patients with unknown primary tumour. A search for prognostic factors in UPT. Anticancer Res. 2004;24:297-301.

11. Karavasilis V, Malamou-Mitsi V, Briasoulis E, et al. Angiogenesis in cancer of unknown primary: clinicopathological study of CD34, VEGF and TSP-1. BMC Cancer. 2005;5(1):25. doi:10.1186/14712407-5-25

12. Karavasilis V, Malamou-Mitsi V, Briasoulis E, et al. Matrix metalloproteinases in carcinoma of unknown primary. Cancer. 2005;104 (10):2282-2287. doi:10.1002/cncr.21454

13. Gatalica Z, Xiu J, Swensen J, Vranic S. Comprehensive analysis of cancers of unknown primary for the biomarkers of response to immune checkpoint blockade therapy. Eur $J$ Cancer. 2018;94:179-186. Return to ref 56 in article. doi:10.1016/j. ejca.2018.02.021

14. Pentheroudakis G, Petrakis D, Goussia A, et al. 1450 POSTER immunohistochemical profiling of signalling pathways in Cancer of Unknown Primary (CUP). Eur J Cancer. 2011;47:S184. doi:10.1016/ S0959-8049(11)70943-1

15. Hedley DW, Leary JA, Kirsten F. Metastatic adenocarcinoma of unknown primary site: abnormalities of cellular DNA content and survival. Eur J Cancer Clin Oncol. 1985;21:185-189. doi:10.1016/ 0277-5379(85)90171-3 
16. Natoli C, Ramazzotti V, Nappi O, et al. Unknown primary tumors Biochim Biophys Acta. 2011;1816(1):13-24.

17. Pantou D, Tsarouha H, Papadopoulou A, et al. Cytogenetic profile of unknown primary tumors: clues for their pathogenesis and clinical management. Neoplasia. 2003;5:23-31. doi:10.1016/S1476-5586(03) 80014-3

18. Wang Y, He SS, Bao Y, et al. Cervical lymph node carcinoma metastasis from unknown primary site: a retrospective analysis of 154 patients. Cancer Med. 2018;7(5):1852-1859. doi:10.1002/cam4.1458

19. Jones W, Allardice G, Scott I, Oien K, Brewster D, Morrison SD. Cancers of unknown primary diagnosed during hospitalization: a population-based study. BMC Cancer. 2017;17:85. doi:10.1186/ s12885-017-3083-1

20. Qaseem A, Usman N, Jayaraj JS, Janapala RN, Kashif T. Cancer of unknown primary: a review on clinical guidelines in the development and targeted management of patients with the unknown primary site. Cureus. 2019;11(9):e5552. Published 2019 Sep 2. doi:10.7759/ cureus. 5552

21. Ang K, Harris J, Wheeler R, et al. Human papillomavirus and survival of patients with oropharyngeal cancer. $N$ Engl $J$ Med. 2010;363(1):24-35. doi:10.1056/NEJMoa0912217

22. Ligey A, Gentil J, Créhange G, et al. Impact of target volumes and radiation technique on loco-regional control and survival for patients with unilateral cervical lymph node metastases from an unknown primary. Radiother Oncol. 2009;93(3):483-487. PMID: 19892420. doi:10.1016/j.radonc.2009.08.027

23. Jensen DH, Hedback N, Specht L, et al. Human papillomavirus in head and neck squamous cell carcinoma of unknown primary is a common event and a strong predictor of survival. PLoS One. 2014;9(11):e110456. doi:10.1371/journal.pone.0110456

24. Zhu L, Wang N. 18F-fluorodeoxyglucose positron emission tomography-computed tomography as a diagnostic tool in patients with cervical nodal metastases of unknown primary site: a meta-analysis. Surg Oncol. 2013;22:190-194. doi:10.1016/j.suronc.2013.06.002
25. Moller AK, Loft A, Berthelsen AK, et al. A prospective comparison of 18F-FDG PET/CT and CT as diagnostic tools to identify the primary tumor site in patients with extracervical carcinoma of unknown primary site. Oncologist. 2012;17:1146-1154. doi:10.16 34/theoncologist.2011-0449

26. Maghami E, Ismaila N, Alvarez A, et al. Diagnosis and management of squamous cell carcinoma of unknown primary in the head and neck: ASCO guideline. J Clin Oncol. 2020;22:2570-2596. doi:10.12 00/JCO.20.00275

27. Binder C, Matthes KL, Korol D, Rohrmann S, Moch H. Cancer of unknown primary-epidemiological trends and relevance of comprehensive genomic profiling. Cancer Med. 2018;7 (9):4814-4824. PMID: 30019510; PMCID: PMC6144156. doi:10. $1002 / \mathrm{cam} 4.1689$

28. Al Kadah B, Papaspyrou G, Linxweiler M, et al. Cancer of unknown primary (CUP) of the head and neck: retrospective analysis of 81 patients. Eur Arch Otorhinolaryngol. 2017;274(6):2. doi:10.1007/ s00405-017-4525-8

29. Hainsworth JD, Spigel DR, Thompson DS, et al. Paclitaxel/carboplatin plus bevacizumab/erlotinib in the first-line treatment of patients with carcinoma of unknown primary site. Oncologist. 2009;14(12):1189-1197. doi:10.1634/theoncologist.2009-0112

30. Krämer A, Hübner G, Schneeweiss A, Folprecht G, Neben K. Carcinoma of unknown primary - an orphan disease? Breast Care. 2008;3(3):164-170. doi:10.1159/000136001

31. Grau C, Johansen LV, Jakobsen J, Geertsen P, Andersen E, Jensen BB. Cervical lymph node metastases from unknown primary tumours: results from a national survey by the Danish society for head and neck oncology. Radiother Oncol. 2000;55:121-129. doi:10.1016/S0167-8140(00)00172-9

\section{Publish your work in this journal}

Cancer Management and Research is an international, peer-reviewed open access journal focusing on cancer research and the optimal use of preventative and integrated treatment interventions to achieve improved outcomes, enhanced survival and quality of life for the cancer patient.
The manuscript management system is completely online and includes a very quick and fair peer-review system, which is all easy to use. Visit http://www.dovepress.com/testimonials.php to read real quotes from published authors. 